\title{
Mosaicos clonais de eucalyptus no planejamento florestal e seus efeitos econômicos e produtivos
}

\author{
Eucalyptus clonal mosaics in forest planning and their \\ effects on wood production and economy
}

\author{
Tayrine Vieira Martins' ${ }^{1}$, Lucas Rezende Gomide ${ }^{2}$, Antônio Carlos Ferraz Filho3, \\ Pedro Resende Silva ${ }^{4}$ e Lucas Amaral de Melo
}

\begin{abstract}
Resumo
Atualmente um novo conceito de planejamento florestal vem se popularizando, o planejamento florestal espacial. Nele, a unidade de manejo ou talhão passa a ser vista de maneira mais abrangente com análise para seu tamanho, forma e distribuição em relação aos demais. Para este problema, foram elaborados modelos de Programação Linear Inteira (PLI), com uso de restrições do planejamento tradicional, restrições espaciais do tipo URM (Unit Restriction Model) colheita e restrição de adjacência clonal, nova aplicação de URM. Objetivou-se, com este trabalho, criar um modelo de minimização de custo utilizando restrição de adjacência clonal. A intenção desta restrição é reduzir o tamanho de áreas homogêneas, em florestas de eucalipto e, desta forma, diminuir a proliferação de pragas e doenças além de contribuir para a tomada de decisão do gestor florestal. O processamento dos dados foi realizado, nos princípios da PLI e gerou 11 cenários que diferenciaram entre si pela natureza das restrições, espacial e não espacial, bem como restrições volumétricas e limite de área a ser reformada. $O$ volume médio colhido nos cenários sem restrição espacial foi $0,64 \%$ maior em relação àqueles com restrições espaciais. A adição de restrição espacial reduziu o valor da função objetivo. Nos cenários, em que houve restrição de área mínima de reforma, houve diferenças significativas nos custos. Para os cenários com $50 \%, 40 \%$ e $30 \%$ de área de reforma, houve um aumento de $15 \%, 11 \%$ e $8 \%$ no custo final, respectivamente. Os mapas gerados evidenciam, na matriz paisagística, a eficácia da aplicação das restrições espaciais. A aplicação da restrição de adjacência clonal mostrou-se melhor que a URM colheita e seu custo ficou próximo do planejamento tradicional, apenas $0,14 \%$ superior, comprovando a viabilidade de sua aplicação no contexto de medida preventiva de proliferação de pragas e doenças, despontando como uma ferramenta potencial para redução de custos com manejo de pragas.
\end{abstract}

Palavras-chave: Planejamento espacial; URM; Matriz paisagística; Pragas florestais.

\begin{abstract}
Currently a new concept of forest planning has become popular, spatial forest planning. In it, the stand is seen as part of a whole and it is considered its size, shape and distribution in relation to the others. For this problem, we used constraints of traditional planning, spatial constraints of the URM (Unit Restriction Model) type and restrictions of clonal adjacency, a new application of URM in forestry. This study aimed to create a cost minimization model using clonal material adjacent spatial restriction. This was done with the intention of reducing the size of homogenous areas in eucalypt forests and thus hinder the proliferation of pests and diseases, as well as contribute to the decision making of the forest manager. Data processing was carried out with the principles of Integer Linear Programming and generated 11 scenarios differentiated by the nature of the restrictions, spatial and non-spatial, as well volumetric restrictions and limitation of area to be reformed. The average volume harvested in the scenarios without space restriction was $0.64 \%$ higher than those with space constraints. The spatial restriction addition reduced the value of the objective function. In the scenarios where there was restriction of a minimum reform area there were significant differences in costs. For scenarios with $50 \%, 40 \%$ and $30 \%$ reform area there was an increase of $15 \%, 11 \%$ and $8 \%$ in the final cost, respectively. The generated maps demonstrated the affective of the application of spatial

\footnotetext{
${ }^{1}$ Mestranda em Engenharia Florestal. UFLA - Universidade Federal de Lavras / Departamento de Ciências Florestais. Campus Universitário - Caixa-postal: 3037 - 37200-000 - Lavras, MG, Brasil. E-mail: tatavmartins@gmail.com.

2Professor Adjunto do Departamento de Ciências Florestais. UFLA - Universidade Federal de Lavras. Campus Universitário - Caixa-postal: 3037 - 37200-000 - Lavras, MG, Brasil. E-mail: lucasgomide@dcf.ufla.br; lucas.amaral@dcf.ufla.br.

3Professor Adjunto do Campus Professora Cinobelina Elvas. UFPI - Universidade Federal do Piauí. Avenida Manoel Gracindo - Km 01 - 64900000 - Bom Jesus, PI, Brasil. E-mail: acferrazfilho@ufpi.edu.br.

${ }^{4}$ Mestre em Engenharia Florestal. UFLA - Universidade Federal de Lavras / Departamento de Ciências Florestais. Campus Universitário - Caixa-postal: 3037 - 37200-000 - Lavras, MG, Brasil. E-mail: pedro.resende@queirozgalvao.com.
} 
constraints in the landscape matrix. The application of restriction of clonal adjacency by the URM proved to be better than traditional harvest URM and its cost was close to the traditional planning, only $0.14 \%$ greater, proving the feasibility of its application in the context of a preventive measure of pest and disease proliferation, emerging as a potential tool to reduce cost of pest management.

Keywords: Spatial planning; URM; Landscape matrix; Forest pests.

\section{INTRODUÇÃO}

O planejamento florestal abrange três características que, em conjunto, tornam o seu exercício um grande desafio para o gestor florestal. Primeiro, a existência de múltiplos objetivos que, em alguns momentos, podem ser conflitantes. Segundo, o planejamento florestal trabalha com períodos longos de tempo, a fim de refletir a natureza dos problemas florestais. E, por fim, o planejamento florestal é complexo em razão do elevado volume de informações requisitadas. Estas dificuldades surgem da existência de interação de muitos processos diferentes, grandes áreas e dados inconsistentes. Geralmente há um alto grau de incerteza quando se prevê o resultado de diferentes variáveis econômicas e ecológicas (DAVIS et al., 2001).

Por um longo tempo o planejamento florestal preocupava-se apenas com aspectos econômicos e de produção. Hoje, há uma maior consciência da importância do padrão paisagístico sobre as funções ecológicas das florestas. Estas preocupações exigem novos desenvolvimentos de modelos, no planejamento florestal, sendo necessário o envolvimento de informações espaciais (DONG et al., 2015).

No planejamento tradicional, o talhão é apenas uma unidade de produção, desconectada dos demais talhões, já, no planejamento espacial, passa-se a observar o tamanho, a forma e a distribuição do talhão no interior da floresta. Baskent e Keles (2005) asseguram que, para este último caso, são formuladas restrições espaciais, sendo as mais conhecidas, restrição de adjacência do tipo URM (unit restriction model) e do tipo ARM (area restriction model). A abordagem URM não permite a colheita de talhões vizinhos em um mesmo período do horizonte de planejamento (HP). A restrição do tipo ARM permite o corte de talhões adjacentes desde que a área a ser colhida não ultrapasse o limite máximo de área permitido. Do ponto de vista do cálculo, a restrição ARM é de difícil formulação, em razão da complexidade de se relacionar os talhões de forma contínua (BACHMATIUK et al. 2015). Para o presente trabalho, aquela que melhor se adéqua ao problema é a do tipo URM, por este motivo ela foi utilizada.

Borges et al. (2014a) defendem que o uso de restrição de adjacência tornou-se uma parte importante nas práticas florestais, em todo o mundo. Diversos trabalhos têm sido realizados com uso de restrições de adjacência, em diferentes contextos, tais como: silvicultura (BORGES et al, 2014b; DONG et al., 2015; SHAN et al., 2009), manutenção de corredores ecológicos (FISCHER; CHURCH, 2003; HEINONEN et al., 2007), controle de área mínima e máxima (MARTINS et al., 2014; MCDILL et al., 2002), desenvolvimento e manutenção de habitat (PUKKALA et al., 2012; TÓTH; MCDILL, 2008), diminuição da fragmentação florestal (BORGES; HOGANSON; 2000; OHMAN; LAMAS, 2005), green up (BOSTON; BETTINGER, 1999; STRIMBU et al., 2010; ZHU; BEETINGER, 2008), dentre outros. No Brasil, alguns trabalhos envolvendo planejamento espacial também vêm sendo realizados, como: Alonso (2003), Binotti et al. (2014), Castro, (2007). No entanto, até o presente momento, não foram encontrados trabalhos envolvendo o uso de restrições espaciais no contexto de proteção e segurança de florestas plantadas contra pragas e doenças.

Considera-se que a cultura do eucalipto é uma das mais importantes do Brasil. No entanto, sofre com ataques de pragas e doenças, desde a sua fase de viveiro até plantios adultos (TUMURA et al., 2012), devido ao uso de clones que são plantados em larga escala comercial. Com o advento e utilização em grande escala da clonagem na silvicultura de eucalipto, a partir da década de 1970, grandes extensões de terra foram ocupadas por um ou poucos materiais genéticos, tornando um risco à ocorrência de pragas e doenças, devido à redução da variabilidade genética.

A busca por plantas resistentes ou tolerantes ao ataque de insetos tem sido uma alternativa economicamente viável para conter as populações das pragas em níveis inferiores ao de dano econômico (QUEIROZ et al., 2013). Alguns desses danos são mais recorrentes e atribuídos à ferrugem (SILVA et al., 2013), lagartas (ZANUNCIO, 1993), besouro amarelo (MAFIA; MENDES, 2014), per- 
cevejo bronzeado (SAAVEDRA et al., 2015), vespa da galha (PEREIRA et al., 2014) e psilídeos (BURCKHARD; QUEIROZ, 2012; CAMARGO et al., 2014).

No entanto, o problema relacionado a pragas tem se agravado em decorrência do aumento de áreas plantadas de eucalipto, principalmente, para a produção de celulose e energia (CAMARGO et al., 2014). Desta forma, faz-se necessária a criação de novas alternativas, que podem auxiliar no controle e prevenção dessas pragas e doenças, como a alteração na disposição espacial dos plantios, criando mosaicos clonais.

Este trabalho teve como objetivo apresentar uma nova aplicação da restrição URM em modelos de minimização de custos de produção, utilizando uma nova abordagem na silvicultura, considerando a adjacência clonal uma alternativa de medida preventiva de proliferação de pragas e doenças em plantios de eucalipto. Além disso, gerar diferentes cenários e avaliar os resultados obtidos, com base nos padrões de dispersão dos talhões e também sobre a produção física e econômica proveniente da floresta.

\section{MATERIAL E MÉTODOS}

O trabalho abordou o problema de planejamento florestal tendo como foco a segurança florestal, no que diz respeito à proliferação de pragas e doenças em plantios de eucalipto. Buscou-se a redução de custos de produção, juntamente com o uso da espacialização ótima de clones ou mosaico de clones, com base na aplicação da restrição de adjacência clonal.

O estudo foi desenvolvido em uma fazenda pertencente a uma empresa do setor siderúrgico, localizada na região nordeste do Brasil. A fazenda é formada por 282 talhões, totalizando uma área de 10.051,45 hectares. A empresa possui 35 tipos de materiais genéticos de eucalipto e uma unidade de produção de carvão (UPC). Esta, por sua vez, é composta por 576 fornos circulares e 54 fornos retangulares. O regime de manejo adotado pela empresa é o Energy Wood, isto é, utiliza a madeira para produção de carvão vegetal que abastece a indústria siderúrgica.

No sentido de resolução do problema, previamente, é necessário gerar as alternativas de manejo (AM). Para este problema, as AM são compostas por todas as combinações possíveis entre talhões, momento de corte, silvicultura (reforma ou brotação), material genético e UPC de destino. Quando há a opção de reforma, existe a alternativa de troca de clone (clone 4, clone 5 ou clone 11). Para a troca de clone, consideraram-se os clones mais utilizados pela empresa. O horizonte de planejamento adotado foi de cinco anos, pois se tratam de florestas de curto ciclo de rotação e destinadas para a produção de carvão. Adotou-se idade mínima de corte cinco anos e máxima de nove anos, sendo cada AM possuindo um custo final de produção.

O valor final dos custos foi calculado pelo somatório dos custos descapitalizados de implantação e manutenção da reforma ou custo de implantação e manutenção da brotação, custo da terra, custo de colheita e de transporte da madeira do talhão até a UPC. Neste último caso, o custo de transporte foi adquirido pelo valor do menor custo de deslocamento do talhão para a UPC. O roteamento e o custo de deslocamento do talhão a UPC foram disponibilizados pela empresa. Os custos obtidos foram descapitalizados, para o ano zero, sendo, então, calculado o VPC (Valor Presente dos Custos) (1). Em que: VCP é o valor dos custos descapitalizados para o ano zero; CT é o custo total no ano $t$ e y é a taxa de juros anual aplicada, considerada de $8 \%$ a.a.

$$
V P C=\sum_{t=0}^{H P} \frac{C T_{t}}{(1+y)^{t}}
$$

As projeções de crescimento volumétrico da floresta foram geradas com base no modelo (2), em que Id é a idade em anos e $\beta_{0}$ e $\beta_{1}$ são os parâmetros da equação. Este mesmo modelo foi ajustado por clones e, também, como um modelo genérico para produção geral da fazenda. Os modelos foram gerados com base nos dados de inventário da empresa. Quando não havia correspondência de clone, foi usado o modelo genérico da fazenda.

$$
\mathrm{V}_{\mathrm{i}}=\mathrm{e}^{\left(\beta_{0}+\beta_{1}\left(\frac{1}{\mathrm{II}}\right)\right)} \pm \varepsilon_{\mathrm{i}}
$$

O problema abordado teve como base o modelo proposto por Johnson e Scheuman (1977). Neste caso, em razão das características de manejo florestal, aliado à colheita, empregou-se o uso de variáveis binárias $\{0,1\}$ para as variáveis decisórias. Considerando essa premissa, a função objetivo 
(3) foi desenvolvida, para minimizar o custo total de produção de madeira em unidades monetárias reais $(\mathrm{R} \$)$, envolvendo todas as atividades florestais do empreendimento. Não foi computada a venda da madeira (receita), pois a empresa consome toda a madeira gerada para a produção de carvão vegetal. Em que: $C_{i j}=$ representa o custo de produção total de cada talhão $i$ seguindo a alternativa de manejo $j$, ao longo do horizonte de planejamento. $X_{i j}=$ variável de decisão (binária) relacionada ao talhão $i$, assinalada a $\mathrm{AM} j ; M=$ número total de unidades de talhões $i ; N=$ o número total de AM $j$ aplicada a cada talhão $i$.

$$
\operatorname{Min} Z=\sum_{i=1}^{M} \sum_{j=1}^{N} C_{i j} \cdot X_{i j}
$$

Por causa da necessidade de produção constante e baixa variação nos estoques produtivos, as restrições volumétricas (4) e (5) foram desenvolvidas, em que: $v_{i j}=$ volume produzido no talhão $i$ conforme prescrição da AM $j, V T$ = variável de decisão contínua relacionada ao volume $\left(\mathrm{m}^{3}\right)$ a ser colhido anualmente a cada ano $T$ do horizonte de planejamento $\{1,2, \ldots, 5\}$.

$$
\begin{aligned}
& \sum_{\mathrm{i}=1}^{M} \sum_{\mathrm{j}=1}^{N} \mathrm{v}_{\mathrm{ij}} \cdot \mathrm{X}_{\mathrm{ij}} \geq 1,1 \cdot \mathrm{V}_{\mathrm{T}} ; \forall_{\mathrm{T}} \\
& \sum_{\mathrm{i}=1}^{M} \sum_{\mathrm{j}=1}^{N} \mathrm{v}_{\mathrm{ij}} \cdot \mathrm{X}_{\mathrm{ij}} \leq 1,5 \cdot \mathrm{V}_{\mathrm{T}} ; \forall_{\mathrm{T}}
\end{aligned}
$$

Em virtude do ciclo curto de rotação, características do manejo florestal para a espécie, a restrição (6) foi desenvolvida, para se adotar apenas uma única alternativa de manejo por talhão, evitando seu abandono.

$$
\sum_{j=1}^{N} \mathrm{X}_{\mathrm{ij}}=1 ; \forall_{\mathrm{i}}
$$

Por outro lado, foram utilizadas restrições de área mínima a ser reformada (7), para atender as exigências da empresa na troca de material genético. Em que: $a_{i}=\mathrm{a}$ área do talhão $i$ a ser reformado, $A=$ área total do projeto em hectares e $R=$ a taxa de reforma $\{0,3 ; 0,4$ e 0,5$\}$ estabelecida previamente.

$$
\sum_{i=1}^{M} \sum_{j=1}^{N} a_{i} \cdot X_{i j} \geq R \cdot A ; \forall_{R}
$$

Restrições de não negatividade e binárias, também, foram incluídas para que as características do problema formulado fossem atendidas.

$$
\mathrm{X}_{\mathrm{ij}} \in\{0,1\}
$$

O conjunto de equações citadas compõe a estrutura do modelo base, sem a inclusão de aspectos espaciais, que se pretende avaliar. Logo, para se obter um melhor entendimento de seus efeitos econômicos, foram definidos três grupos de modelos, contendo um conjunto de cenários econômicos e produtivos. No grupo 1 (planejamento tradicional), não foram testadas restrições espaciais (adjacência na colheita ou mosaico clonal), sendo o mesmo composto pelas restrições (4), (5), (6), (7) e (8).

O grupo 2 contemplou, ainda, a restrição de adjacência URM de colheita, na sua forma de aplicação clássica, conforme proposto por Mcdill et al. (2002), sendo a restrição (9) aplicada para impedir o corte de talhões adjacentes, em que: $m_{i k}=$ coeficientes da matriz binária $\{0,1\}$ de adjacência entre talhões, em que $i=k, k=$ número do talhão avaliado na base.

$$
\sum_{\substack{\mathrm{i}=1 \\ \mathrm{i} \neq \mathrm{k}}}^{\mathrm{M}} \mathrm{m}_{\mathrm{ik}} \cdot \mathrm{X}_{\mathrm{i}}^{\mathrm{T}}+\left[\sum_{\substack{\mathrm{i}=1 \\ \mathrm{i} \neq \mathrm{k}}}^{\mathrm{M}} \mathrm{m}_{\mathrm{ik}}\right] \cdot \mathrm{X}_{\mathrm{i}}^{\mathrm{T}} \leq \sum_{\substack{\mathrm{i}=1 \\ \mathrm{i} \neq \mathrm{k}}}^{\mathrm{M}} \mathrm{m}_{\mathrm{ik}} ; \forall_{\mathrm{k}}, \forall_{\mathrm{T}}, \mathrm{j} \subset \mathrm{T}
$$

Em razão da rigidez causada por este tipo de restrição (GOMIDE et al, 2013), a equação (6) foi flexibilizada, permitindo o abandono de talhões como descrito em (10).

$$
\sum_{j=1}^{N} \mathrm{X}_{\mathrm{ij}} \leq 1 ; \forall_{\mathrm{i}}
$$

O grupo 3 considera o uso de restrições de adjacência clonal (mosaico clonal) e as equações bases de restrições do modelo (4), (5), (6), (7) e (8). Contudo, quando houver a opção de reforma, ou seja, novos plantios na área, há uma preocupação com a troca de material genético (clones 4, 5 ou 11), que não poderão formar blocos de talhões homogêneos e vizinhos de um mesmo material 
genético, gerando um mosaico clonal. A restrição de adjacência clonal (11) poderá funcionar como um método preventivo ou barreira na proliferação de pragas e doenças, auxiliando na segurança e proteção dos plantios. Em que, $c=$ marcador que indica o clone $c=\{4,5,11\}$.

$$
\sum_{\substack{\mathrm{i}=1 \\ \mathrm{i} \neq \mathrm{k}}}^{\mathrm{M}} \mathrm{m}_{\mathrm{ik}} \cdot \mathrm{X}_{\overline{\mathrm{i}}}^{\mathrm{Tc}}+\left[\sum_{\substack{\mathrm{i}=1 \\ \mathrm{i} \neq \mathrm{k}}}^{\mathrm{M}} \mathrm{m}_{\mathrm{ik}}\right] \cdot \mathrm{X}_{\overline{\mathrm{T}}}^{\mathrm{Tc}} \leq \sum_{\substack{\mathrm{i}=1 \\ \mathrm{i} \neq \mathrm{k}}}^{\mathrm{M}} \mathrm{m}_{\mathrm{ik}} ; \forall_{\mathrm{c}}, \forall_{\mathrm{k}}, \forall_{\mathrm{T}}, \mathrm{j} \subset \mathrm{T}
$$

A construção de cenários fez-se necessária, uma vez que amplia a possibilidade de discussão, auxiliando na tomada de decisão e avalição de seus impactos econômicos e produtivos. Ao todo foram gerados 11 cenários, variando as taxas de reforma e a combinação de restrições para a sua formação (Tabela 1). A formulação dos problemas de programação linear inteira foi realizada no software LINGO $^{\oplus}$ (LINDO SystemsInc), versão 15.0 de licença acadêmica, empregando o algoritmo branch and bound.

Tabela 1. Combinação de cenários por grupo considerando restrições volumétricas, binária, abandono e não abandono, área mínima de reforma, URM colheita e restrição clonal.

Table 1. Combination of group scenarios with consideration of the following restrictions: volumetric; binary; abandonment and non-abandonment; minimum reform area; harvesting and clonal constraint URM.

\begin{tabular}{|c|c|c|c|c|c|c|c|c|c|c|c|}
\hline \multirow{3}{*}{ Equações } & \multicolumn{11}{|c|}{ Cenários } \\
\hline & \multicolumn{4}{|c|}{ Grupo 1} & \multicolumn{3}{|c|}{ Grupo 2} & \multicolumn{4}{|c|}{ Grupo 3} \\
\hline & 1.1 & 1.2 & 1.3 & 1.4 & 2.1 & 2.2 & 2.3 & 3.1 & 3.2 & 3.3 & 3.4 \\
\hline 3 & $x$ & $x$ & $x$ & $x$ & $x$ & $x$ & $x$ & $x$ & $x$ & $x$ & $\mathrm{x}$ \\
\hline 4 & $x$ & $x$ & $x$ & $x$ & $x$ & $x$ & $x$ & $x$ & $x$ & $x$ & $x$ \\
\hline 5 & $x$ & $x$ & $x$ & $x$ & $x$ & $x$ & $x$ & $x$ & $x$ & $x$ & $x$ \\
\hline 6 & $x$ & $x$ & $x$ & $x$ & & & & $x$ & $x$ & $x$ & $x$ \\
\hline 71 & & $x$ & & & $x$ & & & & $x$ & & \\
\hline 72 & & & $x$ & & & $x$ & & & & $x$ & \\
\hline 73 & & & & $x$ & & & $x$ & & & & $x$ \\
\hline 8 & $x$ & $x$ & $x$ & $x$ & $x$ & $x$ & $x$ & $x$ & $x$ & $x$ & $x$ \\
\hline 9 & & & & & $x$ & $x$ & $x$ & & & & \\
\hline 10 & & & & & $x$ & $x$ & $x$ & & & & \\
\hline 11 & & & & & & & & $x$ & $x$ & $x$ & $x$ \\
\hline
\end{tabular}

Onde: I = taxa de reforma $50 \%, 2$ = taxa de reforma $40 \%$ e $3=$ taxa de reforma $30 \%$

\section{RESULTADOS E DISCUSSÃO}

A combinação das possibilidades de manejo gerou um total de 3.536 variáveis de decisão, sendo o resultado do processamento dos cenários, pelo método exato, é apresentado de maneira sumarizada, na Tabela 2.

Tabela 2. Resultado de custo, tempo de processamento, volume e número de talhões colhidos.

Table 2. Results of cost, processing time, volume and number of harvested stands.

\begin{tabular}{|c|c|c|c|c|c|c|}
\hline Grupo & Cenário & Reforma (\%) & Custo (R\$) & $\begin{array}{c}\text { Tempo de } \\
\text { processamento (s) }\end{array}$ & $\begin{array}{c}\text { Volume total } \\
\qquad\left(\mathrm{m}^{3}\right)\end{array}$ & $\begin{array}{c}\text { Número de talhões } \\
\text { colhidos }\end{array}$ \\
\hline \multirow{4}{*}{1} & 1.1 & - & $55.523 .444,59$ & 102 & $319.378,90$ & 282 \\
\hline & 1.2 & 50 & $63.848 .048,27$ & 105 & $319.381,40$ & 282 \\
\hline & 1.3 & 40 & $61.819 .070,62$ & 106 & $319.381,50$ & 282 \\
\hline & 1.4 & 30 & $59.971 .319,54$ & 52 & $319.381,60$ & 282 \\
\hline \multirow{3}{*}{2} & 2.1 & 50 & $36.530 .633,18$ & 19.692 & $185.462,60$ & 136 \\
\hline & 2.2 & 40 & $28.534 .293,18$ & 324.000 & $143.116,90$ & 106 \\
\hline & 2.3 & 30 & $20.669 .438,06$ & 324.000 & $113.175,50$ & 81 \\
\hline \multirow{4}{*}{3} & 3.1 & - & $55.648 .790,31$ & 16.620 & $317.327,90$ & 282 \\
\hline & 3.2 & 50 & 63.917.696,35 & 64.426 & $317.327,90$ & 282 \\
\hline & 3.3 & 40 & $61.895 .773,40$ & 3.455 & $317.327,90$ & 282 \\
\hline & 3.4 & 30 & $60.044 .865,60$ & 1.938 & $317.327,90$ & 282 \\
\hline
\end{tabular}


Os cenários, contendo as restrições de adjacência, foram mais complexos de serem formulados, exigindo, ainda, um maior esforço computacional para a obtenção de soluções ótimas. Essa dificuldade pode ser justificada pela complexidade em se identificar as relações de adjacência entre talhões, a partir de uma combinação ótima como solução. Além disso, esses modelos continham um número maior de restrições. No trabalho de Tóth et al. (2012), foi evidenciado que a estrutura espacial do problema pode exercer impacto negativo no tempo, uma vez que se eleva o número de combinações, deixando a formulação mais complexa. Embora o uso das restrições espaciais aumente a complexidade do problema, Borges et al. (2014b) defendem que, para ter ferramentas eficientes de planejamento, o uso de restrições espaciais é imprescindível. Em outro trabalho, Borges et al. (2014b) afirmam que as restrições de adjacência, juntamente com as restrições de volume de colheita, são importantes para o manejo e gestão da floresta.

No processamento dos modelos, o método exato de resolução contribuiu para um elevado tempo de processamento, que variou de 35 a 324.000 segundos (Tabela 2). O mesmo comportamento foi observado nos trabalhos de Bettinger (2009), Gomide et al. (2010), Tóth et al. (2012), Bachmatiuk et al. (2015) e Dong et al. (2015). A estratégia a ser seguida, para reduzir este tempo, pode estar associada a métodos aproximativos, como as meta-heurísticas, observados nos trabalhos de Öhman e Eriksson (2002) e Pukkala e Kurttila (2005).

Quando se analisa o número de talhões abandonados por cenário, constatou-se que, nos cenários do grupo 2, seu valor foi extremamente alto, contendo valores de $51 \%$ a $71 \%$ de talhões abandonados. Nesse grupo, foi necessário permitir o abandono de talhões no modelo, para encontrar soluções viáveis. Isso demonstra a rigidez dessas restrições e gastos em tempo de processamento. Além disso, surge a necessidade de se avançar em métodos aproximativos, com o uso de meta-heurísticas, conforme apresentado por Ohman e Lamas (2005) e Pukkala e Heinonen (2006).

Bertomeu e Romero (2001) e Murray e Weintraub (2002) acreditam que o uso do método exato, em problemas complexos e extensos, pode ser pouco realista. Esses mesmos autores sugerem a busca por alternativas de solução, quando os métodos de programação matemática exata tornam-se impraticáveis. Eles sugerem a utilização de métodos heurísticos, que são processos de pesquisa, aplicam-se estratégias e regras que oferecem resultados satisfatórios em um tempo computacional razoável. No entanto, não existe garantia de que a melhor solução encontrada seja a solução ótima global (BACHMATUK; et al 2015).

É evidente que, a depender das exigências do modelo, haverá a possibilidade de abandono de talhões e, com isso, uma redução no volume de madeira colhido, como ocorrido nos cenários do grupo 2 (Figura 1). Os efeitos volumétricos foram visíveis, quando empregada a restrição URM, para a colheita em seu conceito puro. Porém, um comportamento oposto foi observado considerando a restrição de adjacência clonal (mosaico clonal), o que evidencia a grande oportunidade de se formar uma paisagem mais resistente ao ataque de pragas, sem prejuízos às metas volumétricas.

A adição de restrições espaciais, grupo 3, aumentou o valor da função objetivo, ou seja, aumentando o custo total, exceto para os cenários grupo 2, já que se permitiu o abandono de talhões. $\mathrm{O}$ impacto maior foi entre os cenários com reforma. Nesses, foram constatados diferenças entre os valores da função objetivo, ao se comprar os cenários do grupo 3 e grupo 1. Como esperado, a restrição de reforma influenciou no custo final, aumentando proporcionalmente com seu incremento. No grupo 3, quando comparado o cenário 3.1 (sem reforma) com os que houveram reforma 3.2 (50\%), 3.3 (40\%) e $3.4(30 \%)$, percebeu-se um aumento de 15\%, 11\% e 8\% no custo final, respectivamente. Contudo, a diferença entre as médias dos custos dos cenários do grupo 1 e 3 foi de apenas $0,14 \%$ e $0,64 \%$ para o volume total produzido.

Nos trabalhos de Binoti et al. (2014) e Dong et al. (2015), a adição de restrição espacial reduziu o valor da função objetivo. Gomide et al. (2010), em seu trabalho, observou que a incorporação de restrições do tipo URM, também, reduziu a função objetivo, em 3,74\%. Para este estudo, essa diferença não foi expressiva, a restrição de adjacência aplicada, no controle espacial dos clones, apresentou valores entre $0,12 \%$ a 0,23\%, no grupo 3 . O efeito da reforma não influenciou a restrição de adjacência clonal, uma vez que os aumentos dos custos finais seguiram a mesma tendência, para os três grupos, o que demonstra a viabilidade desta aplicação. Contudo, este fator deve ser ponderado no empreendimento. 
Na Figura 1 observa-se o comportamento do volume produzido anualmente por cenário testado. Assim, pode-se comparar os cenários sem imposição de restrições de reforma de área (Figura 1a), com os cenários envolvendo reforma de área mínima de 50\%, 40\% e 30\%, conforme disposto nas figuras $1 \mathrm{~b}$, 1c e $1 \mathrm{~d}$, respectivamente.
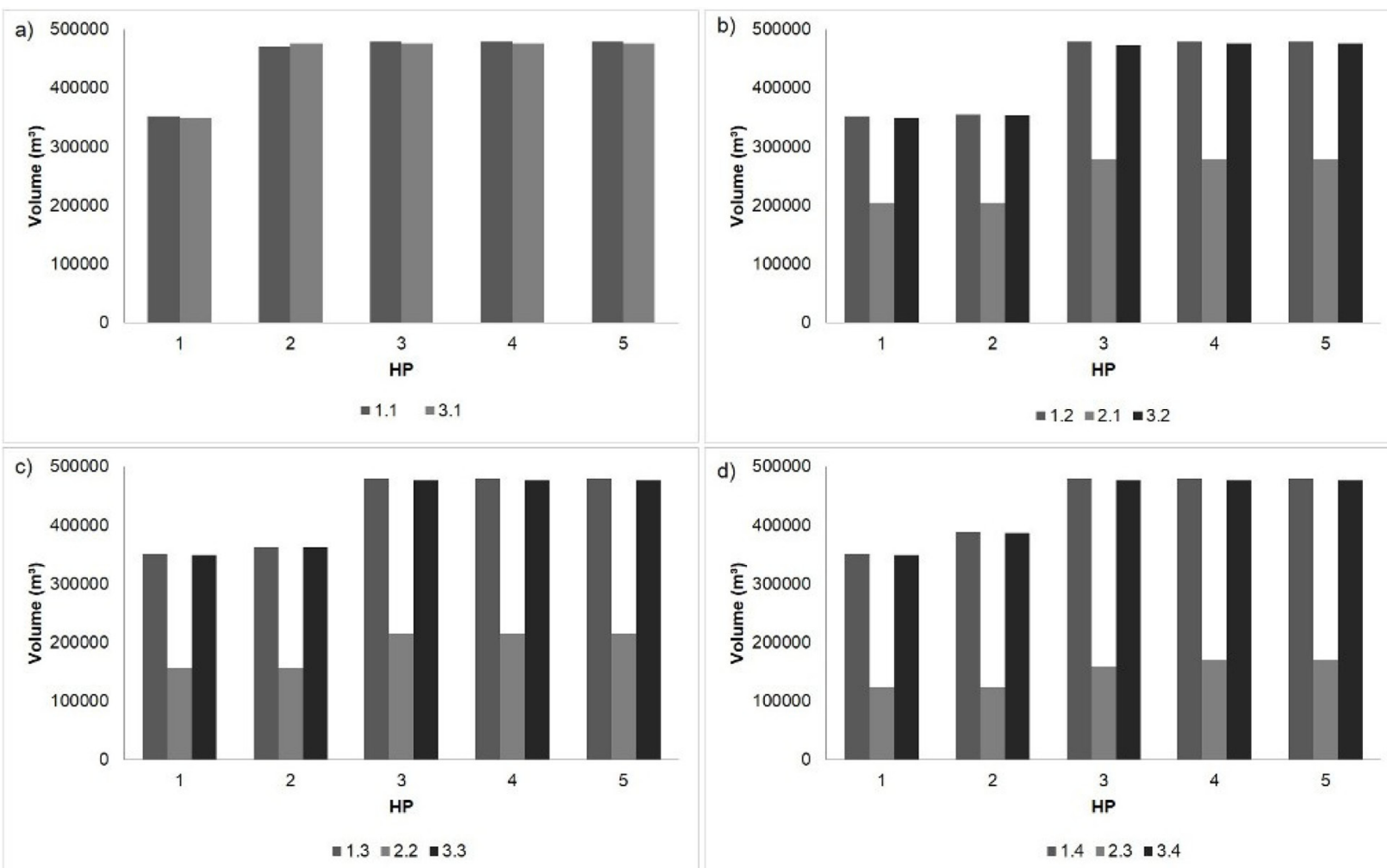

Figura 1. Gráfico de volume para cada cenário ao longo do horizonte de planejamento. Cenário sem área de reforma (a), com $50 \%$ de área de reforma (b), com $40 \%$ de área de reforma (c) e com 30\% de área de reforma (d).

Figure 1. Volume chart for each scenario throughout the planning horizon. Scenario without reform area (a), 50\% of reform area (b), with $40 \%$ of reform area (c) and $30 \%$ of reform area (d).

O volume médio, colhido sem restrição espacial nos cenários (grupo 1), foi, em média, 0,64\% superior, quando comparado com o grupo 3. No trabalho de Dong et al. (2015), não houve grandes diferenças de volume colhido, assim como o observado no presente estudo, porém sua aplicação ficou restrita à adjacência direcionada para a colheita florestal. Fica evidente e complementar que a intensidade mínima de área de reforma pouco influenciou o volume colhido ao longo do HP. Contudo, neste caso, esses efeitos podem ser influenciados mais pelos modelos de crescimento e produção empregados, do que, realmente, uma solução no sentido combinatório, já que a qualidade do inventário florestal contínuo é um ponto importante na gestão das florestas.

Na maioria dos cenários, verificou-se que o volume colhido, ao longo do HP, tornou-se constante a partir do terceiro ano do HP. Isso significa que a floresta caminha para a regulação e, neste caso, a floresta apresenta uma tendência de idade média próxima dos três anos de idade, o que disponibiliza uma maior volumetria a partir do terceiro ano do horizonte de planejamento. Segundo Leuschner (1984), floresta regulada é aquela em que há produções anuais ou periódicas de igual volume, tamanho ou qualidade, também, sendo associada ao compartimento em área colhido anualmente. Verifica-se que nenhuma floresta se torna totalmente regulada, em razão de aspectos econômicos de macro e microeconomia, como, ainda, ligados às questões de mercado.

A visualização do mosaico clonal pode ser vista nos mapas de agendamento da colheita florestal, a partir das saídas apresentadas pelos modelos em cada cenário. Os mapas foram fundamentais para avaliar a estrutura na matriz paisagística. A Figura 2 comprova a eficácia da espacialização dos clones pelo modelo matemático. 


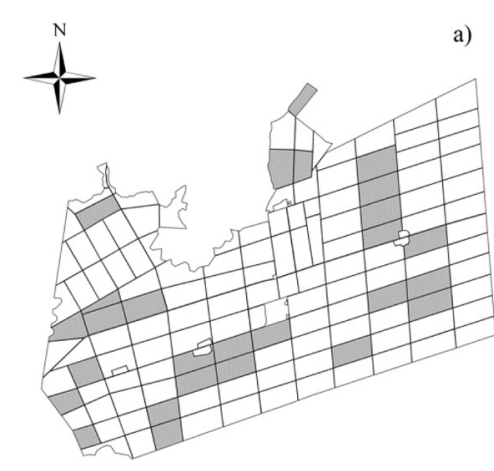

Legenda

Talhões para corte

Horizonte Planejamento 4

Clones

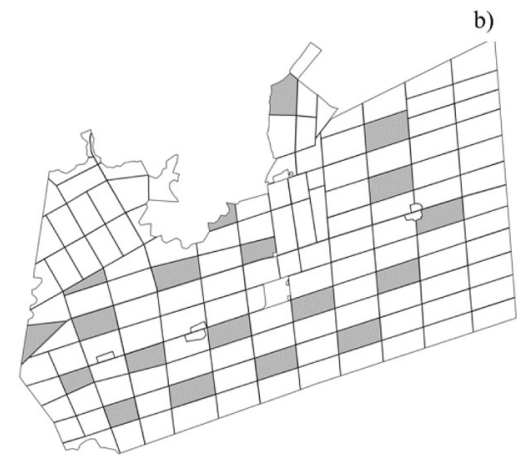

Talhões para corte

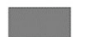

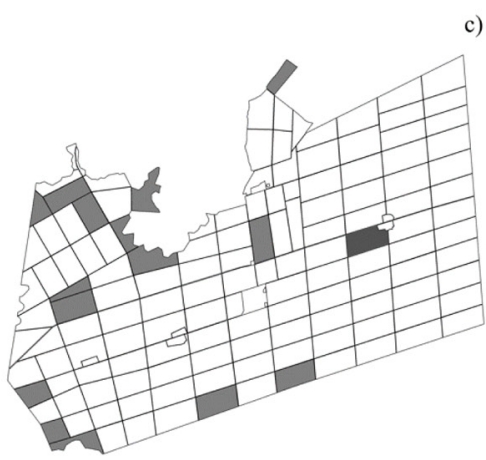

\begin{tabular}{lllll}
0 & 1,25 & 2,5 & 5 & 7,5 \\
\hline
\end{tabular}

Figura 2. Mapa parcial da fazenda com ilustração do efeito espacial no agendamento da colheita florestal para um único ano do horizonte de planejamento, sendo: (a) cenário 1.2, (b) cenário 2.2 e (c) cenário 3.2.

Figure 2. Partial map of the farm illustrating the spatial effect on the forest harvesting schedule for a single year of the planning horizon, being: (a) scenario 1.2, (b) scenario 2.2 and (c) scenario 3.2.

Assim, ficam claras as diferenças entre a Figura 2a, 2b e 2c. Na Figura 2a, não há restrições espaciais e, desta forma, os talhões são colhidos com base apenas no planejamento tradicional. Nesse caso, áreas contíguas são colhidas, permitindo a reforma de áreas adjacentes com clones semelhantes. Por sua vez, na Figura 2b, os talhões adjacentes não são colhidos no mesmo período do horizonte de planejamento, mas essa estratégia causou impactos na produção volumétrica. Por fim, na Figura 2c, observa-se o mosaico clonal, que controla o plantio do mesmo material genético adjacente, para o mesmo ano, tendo como consequência a redução de extensas áreas de plantio para um mesmo clone.

A proposta dessa ideia de mosaico clonal deriva de estudos que trabalham com o conceito de green up (STRIMBU et al., 2010; ZHU; BEETINGER, 2008), amplamente discutidos na literatura e com destaques. As restrições de adjacência do tipo URM são utilizadas em problemas de green up. Nesses casos, visam a questões relacionadas a impactos visuais sobre a paisagem, de forma exclusiva e, por conseguinte, à proteção e conservação das florestas.

O presente trabalho propôs uma nova aplicação deste tipo de restrição, que poderá auxiliar na construção de paisagens mais resistentes à propagação de doenças e patógenos. Por outro lado, o crescimento da eucaliptocultura (monocultura) é acompanhado com o aumento de ataques de pragas e doenças em plantios, assim, estratégias como a implementada neste estudo mostraram-se aplicáveis. Ferreira (1989) recomendou evitar plantios, em larga escala, de procedências muito suscetíveis, para evitar o ataque contínuo e devastador de pragas e doenças. Como exemplo, a propagação da ferrugem (Puccinia psidii) que no Brasil, constitui uma das principais doenças da cultura do eucalipto (ALFENAS et al., 1989; FERREIRA, 1989), assim como o ataque de Glycaspis brimblecombei (Moore, 1964) (BRENNAN; WEINBAUM, 2001), Costalimaita ferruginea (Fabricius, 1801) e Costalimaita lurida (Lefévre, 1891) (MAFIA; MENDES, 2014), Thaumastocoris peregrinus (MUTITU et al., 2013) e lagartas desfolhadoras (Lepidópteras) (BITTENCOURT; BERTI FILHO, 2004).

De maneira geral, pragas e doenças do eucalipto podem causar perdas expressivas, em toda a cadeia produtiva, provocando reduções no crescimento e na produtividade, ou mesmo, a morte de mudas nos viveiros e de plantas no campo (MAFIA; MENDES, 2014). Todavia, estudos envolvendo o custo dessas perdas, bem como a possibilidade de se verificar a real eficiência da proposta no campo, ainda, são escassos, carecendo, portanto de mais estudos. Um fato que pode gerar maior confiança é o aumento no número de clones a serem cultivados, propiciando maior resistência desses povoamentos. Espera-se que um número maior de clones cultivados, como opção de plantio no modelo, promova um menor impacto no valor da função objetivo e nos resultados volumétricos produzidos, uma vez que aumentam as opções viáveis para a solução ótima final. 


\section{CONCLUSÕES}

O uso da restrição de adjacência clonal é uma opção viável para gerar mosaicos com maior heterogeneidade de clones. As perdas volumétricas nos cenários, com esta restrição, foram reduzidas e abaixo de $1 \%$, quando comparado com o planejamento tradicional. O efeito do custo total seguiu a mesma tendência, sendo superior em apenas $0,14 \%$, em média. O maior valor gasto poderá ser revertido, com economias no controle de pragas e doenças, mas esta afirmativa que carece de estudos aplicados em campo.

\section{REFERÊNCIAS BIBLIOGRÁFICAS}

ALFENAS, A. C.; DEMUNER, N. L.; BARBOSA, M. M. A ferrugem e as opções de controle. Correio Agrícola, São Paulo, n. 1, p. 18-20, 1989.

ALONSO, L. R. L. O problema da consideração de restrições de adjacência em um planejamento florestal. 2003. 114 p. Dissertação (Mestrado em Métodos Numéricos em Engenharia) - Universidade Federal do Paraná, Curitiba. 2003.

BACHMATIUK, J.; GARCIA-GONZALO, J.; BORGES, J. G. Analysis of the performance of different implementations of a heuristic method to optimize forest harvest scheduling. Silva Fennica, Helsinki, v. 49, n. 4, p. 01-18, 2015.

BASKENT, E. Z.; KELES, S. Spatial forest planning: a review. Ecological Modelling, Amsterdam, v. 188, v. 2/4, p. 145-173, Nov. 2005.

BERTOMEU, M.; ROMERO, C. Managing forest biodiversity: a zero-one goal programming approach. Agricultural Systems, Essex, v. 68, n. 3, p. 197-213, 2001.

BETTINGER, P. A prototype method for integrating spatially-referenced wildfires into a tactical forest planning model. Research Journal of Forestry, Oxford, v. 4, n. 3, p. 158-172, 2010.

BINOTI, D. H. B.; BINOTI, M. L. M.; LEITE, H. G.; GLERIANI, J. M.; RIBEIRO, A. S. Inclusion and effect of spatial characteristics on models of forest regulation. Cerne, Lavras, v. 20, n. 1, p. 157-164, 2014.

BITTENCOURT, M. A. L.; BERTI FILHO, E. Exigências térmicas para o desenvolvimento de Palmistichus elaeisis (Hymenoptera, Eulophidae) em pupas de cinco espécies de lepidópteros. Iheringia, Porto Alegre, v. 94, n. 3, p. 321-323, 2004.

BORGES, P.; BERGSENG, E.; EID, T. Applying simulated annealing using different methods for the neighborhood search in forest planning problems. European Journal of Operational Research, Amsterdam, v. 233, n. 3, p. 700-710, 2014 a.

BORGES, P.; BERGSENG, E.; EID, T. Adjacency constraints in forestry-a simulated annealing approach comparing different candidate solution generators. Mathematical and Computational Forestry \& Natural Resource Sciences, Norwegian, v. 6, n. 1, p. 11, 2014b.

BOSTON, K.; BETTINGER, P. An analysis of Monte Carlo integer programming, simulated annealing, and tabu search heuristics for solving spatial harvest scheduling problems. Forest Science, Bethesda, v. 45, n. 2, p. 292-301, 1999.

BRENNAN, E. B.; WEINBAUM, S. A. Stylet penetration and survival of three psyllid species on adult leaves and 'waxy' and 'de-waxed' juvenile leaves of Eucalyptusglobulus. Entomologia Experimentalis et Applicata, Dordrecht, v. 100, n. 3, p. 355-363, 2001. 
Martins et al. - Mosaicos clonais de eucalyptus no planejamento florestal e seus efeitos econômicos e produtivos

BURCKHARDT, D.; QUEIROZ, D. L. Checklist and comments on the jumping plant-lice (Hemiptera: Psylloidea) from Brazil. Zootaxa, Basel, v. 3571, p. 26-48, 2012.

CAMARGO, J. M. M.; ZANOL, K. M. R.; QUEIROZ, D. L.; DEDECECK, A.; OLIVEIRA, E. B.; MELIDO, R. C. N. Resistência de clones de Eucalyptus ao psilídeo-de-concha. Pesquisa Florestal Brasileira, Colombo, v. 34, n. 77, p. 91-97, 2014.

CASTRO, R. R. Regulação de florestas eqüiâneas incluindo restrições de adjacência. 2007. 64 p. Dissertação (Mestrado em Ciência Florestal) - Universidade Federal de Viçosa, Viçosa, 2007.

DAVIS, L. (Ed.). Forest management: to sustain ecological, economic, and social values. McGraw-Hill College, 2001. 816 p.

DONG, L.; BERTINGER, P.; LIU, Z. QIN, H. A comparison of a neighborhood search technique for forest spatial harvest scheduling problems: a case study of the simulated annealing algorithm. Forest Ecology and Management, Amsterdam, v. 356, p. 124-135, 2015.

FERREIRA, F. A. Ferrugem do eucalipto. In: SIF - SOCIEDADE DE INVESTIGAÇÕES FLORESTAIS. Patologia florestal: principais doenças florestais no Brasil. Viçosa: Sociedade de Investigações Florestais, p. 129-146, 1998.

FISCHER, D. T.; CHURCH, R. L. Clustering and compactness in reserve site selection: an extension of the biodiversity management area selection model. Forest Science, Bethesda, v. 49, n. 4, p. 555-565, 2003.

GOMIDE, L. R.; ARCE, J. E.; SILVA, A. C. L. Comparação entre a meta-heurística Simulated Annealing e a programação linear inteira no agendamento da colheita florestal com restrição de adjacência. Ciência Florestal, Santa Maria, v. 23, n. 2, p. 449-460, 2013.

GOMIDE, L. R.; ARCE, J. E.; SILVA, A. C. L. Efeito das restrições espaciais de adjacência no planejamento florestal otimizado. Floresta, Curitiba, v. 40, n. 3, p. 573-584, 2010.

HEINONEN, T. Developing spatial optimization in forest planning. 2007. 48 p. Dissertation (Master in Agriculture and Forestry) - University of Joensuu, Joensuu, 2007.

JOHNSON, K. N.; SCHEURMANN, H. L. Techiniques for prescribing optimal timber harvest and investment under different objectives- discussion and 76 synthesis. Forest Science, Bethesda, v. 18, n. 1, p. 01-31, 1977.

LEUSCHNER, W. A. Introduction to forest resource management. New York: John Willey \& Sons, 1984.

MAFIA, R. G.; MENDES, J. E. P. Surtos e danos causados pelos besouros desfolhadores Costalimaita ferruginea (Fabricius, 1801) e Costalimaita lurida (Lefévre, 1891) (Coleoptera: Chrysomelidae) em plantios de eucalipto. Revista Árvore, Viçosa, v. 38, n. 5, p. 829-836, 2014.

MARTINS, I.; YE, M.; CONSTANTINO, M.; FONSECA, M. C.; CADIGMA, J. Modeling target volume flows in forest harvest scheduling subject to maximum area restrictions. Top, Madrid, v. 22, n. 1, p. 343-362, 2014.

MCDILL, M. E.; REBAIN, S. A.; BRAZE, J. Harvest Scheduling with Area-Based Adjacency Constraints. Forest Science, Bethesda, v. 48, n. 4, p. 631-642, 2002.

MUTITU, E.; GARNAS, J. R.; HURLEY, B. P.K.; WINGFIELD, M. J.; HARNEY, M.; BUSH, S. J.; SLIPPERS, B. Biology and rearing of Cleruchoides noackae (Hymenoptera: Mymaridae), an egg parasitoid for the biological control of Thaumastocoris peregrinus (Hemiptera: Thaumastocoridae). Journal of Economic Entomology, Oxford, v. 106, n. 5, 1979-1985, 2013.

MURRAY, A. T.; WEINTRAUB, A. Scale and unit specification influences in harvest scheduling with maximum area restrictions. Forest Science, v. 48, n. 4, p. 779-789, 2002. 
ÖHMAN, K.; ERIKSSON, L. O. Allowing for spatial consideration in longterm forest planning by linking linear programming with simulated annealing. Forest Ecology and Management, Amsterdam, v. 161, n. 1/3, p. 221-230, 2002.

ÖHMAN, K.; LÄMÅS, T. Reducing forest fragmentation in long-term forest planning by using the shape index. Forest Ecology and Management, Amsterdam, v. 212, n. 1, p. 346-357, 2005.

PEREIRA, J. M.; MELO, A. P. C.; RODRIGUES, O. D.; DIAS, T. K. R.; WILCKEN, C. F. Registro de Leptocybe invasa no estado de Goiás. Ciência Rural, Santa Maria, v. 44, n. 10, p. 1721-1724, 2014.

PUKKALA, T.; HEINONEN, T. Optimizing heuristic search in forest planning. Nonlinear Analysis: real world applications, Oxford, v. 7, n. 5, p. 1284-1297, 2006.

PUKKALA, T.; KURTTILA, M. Examining the performance of six heuristic search techniques in different forest planning problems. Silva Fennica, Helsinki, v. 39, n. 1, p. 67-80, 2005.

PUKKALA, T.; SULKAVA, R.; JAAKKLOA, L.; LAHDE, E. Relationships between economic profitability and habitat quality of Siberian jay in uneven-aged Norway spruce forest. Forest Ecology and Management, Amsterdam, v. 276, p. 224-230, 2012.

QUEIROZ, D. L.; MAJER, J.; BURCKHARDT, D.; ZANETTI, R.; FERNANDEZ, J. I.; QUEIROZ, E. C.; GARRASTAZU, M.; FERNANDEZ, B.; V.; ANJOS, N. Predicting the geographical distribution of Glycaspis brimblecombei (Hemiptera:Psylloidea) in Brazil. Australian Journal of Entomology, Canberra, v. 52, n. 1, p. 20-30, 2013.

SAAVEDRA, M. C.; WITHERS, T. M.; HOLWELL, G. I. Susceptibility of four Eucalyptus host species for the development of Thaumastocoris peregrinus Carpintero and Dellapé (Hemiptera: Thaumastocoridae). Forest Ecology and Management, Amsterdam, v. 336, p. 210-216, 2015.

SHAN, Y.; BETTINGER, P.; CIESZEWSKI, C. J.; LI, R. T. Trends in spatial forest planning. Mathematical and Computational Forestry \& Natural Resource Sciences, Norwegian, v. 1, n. 2, p. 86-112, 2009.

SILVA, P. H. M.; MIRANDA, A. C.; MORAES, M. L. T.; FURTADO, STAPE, J. L.; ALVARES, C. A.; SENTELHAS, P. C.; MORI, E. S.; SEBBEN, A. M. Selecting for rust (Puccinia psidii) resistance in Eucalyptus grandis in São Paulo State, Brazil. Forest Ecology and Management, Amsterdam, v. 303, p. 91-97, 2013.

STRIMBU, B. M.; INNES, J. L.; STRIMBU, V. F. A deterministic harvest scheduler using perfect bin-packing theorem. European Journal of Forest Research, Amsterdam, v. 129, n. 5, p. 961-974, 2010.

TÓTH, S. F.; MCDILL, M. E.; KONNYU, N.; GEORGE, S. A strengthening procedure for the path formulation of the area-based adjacency problem in harvest scheduling models. Math. Mathematical and Computational Forestry \& Natural Resource Sciences, Norwegian, v. 4, n. 1, p. 27-49, 2012.

TÓTH, S. F.; MCDILL, M. E. Promoting large, compact mature forest patches in harvest scheduling models. Environmental Modeling \& Assessment, Washington, v. 13, n. 1, p. 01-15, 2008.

TUMURA, K. G.; DE PIERI, C.; FURTADO, E. L. Murcha por Ceratocystis em eucalipto: avaliação de resistência e análise epidemiológica. Summa Phytopathologica, Jaguariúna, v. 38, n. 1, p. 54-60, 2012.

ZANUNCIO, J. C.(Org.). Manual de pragas em floresta: biologia, ecologia e controle (1). Viçosa: IPEF, SIF, 140p. 1993.

ZHU, J.; BETTINGER, P. Estimating the effects of adjacency and green-up constraints on landowners of different sizes and spatial arrangements located in the southeastern US. Forest Policy and Economics, Amsterdam, v. 10, n. 5, p. 295-302, 2008.

Recebido em 23/08/2016

Aceito para publicação em 24/07/2017

Sci. For., Piracicaba, v. 45, n. 116, p. 727-737, dez. 2017

DOI: dx.doi.org/10.18671/scifor.v45n116.13 
